\title{
Estudio de clima ético hospitalario en tres instituciones hospitalarias de Bogotá, Colombia.
}

\author{
Study on the ethics situation in three \\ Bogota D.C., Colombian Hospitals
}

MCE. Olga Muñoz*, Mtra. Beatriz Peña Riveros**, Mtra. Gloria Lucía Arango Bayer ${ }^{\star \star *}$

Mtra.Carolina Gutierrez López ${ }^{\star * * *}$ y MCE.Gloria Inés Prieto de Romano*****

*Profesor Titular Facultad de Enfermería, Fundación Universitaria de Ciencias de la Salud, Bogotá, Colombia, ** Profesor Asociado, Facultad de Enfermería, Universidad Nacional de Colombia, Bogotá, Colombia, ***Profesor Asistente, Facultad de Enfermería, Universidad Nacional de Colombia, Bogotá, Colombia, ****Profesor Auxiliar, Facultad de Enfermería, Universidad de la Sabana, Bogotá, Colombia y $* \star \star * \star$ Profesor Titular, Facultad de Enfermería. Pontificia Universidad Javeriana y Fundación Universitaria de Ciencias de la Salud, Bogotá, Colombia.

\section{Resumen}

Con el objetivo de medir el clima ético hospitalario en tres Instituciones Prestadoras de Servicios de Salud - IPS - de Bogotá, según la percepción de las enfermeras se realizó un estudio en dos fases: la primera de ellas orientada a hacer ajustes al instrumento "Percepción del Clima Ético Hospitalario" diseñado por la doctora Linda Olson, con base en las recomendaciones formuladas por Gutiérrez, e introducirle nuevas preguntas, acordes al contexto colombiano, para luego evaluar psicométricamente la validez y confiabilidad del instrumento.

La segunda fase consistió en aplicar dicho instrumento a 219 enfermeras en tres IPS: una privada, una pública y una de un régimen especial.

Los resultados sugieren que el instrumento con las nuevas preguntas registra una validez de contenido que oscila entre 0,66 y 1 y una confiabilidad global de 0.94 con una correlación superior a 0,38 y una validez de constructo donde los 8 primeros factores obtenidos explican el 60,22\% de la varianza total, con una carga mínima para cada ítem de 0,3 . Las preguntas elaboradas por el grupo investigador tuvieron un Alpha de Cronbach de 0,87.
Los resultados demuestran que este instrumento, a nivel global, tiene una alta validez y confiabilidad para medir Clima Ético Hospitalario como herramienta diagnóstica necesaria para la intervención sobre esta temática y el clima organizacional en general en las instituciones hospitalarias.

La aplicación del instrumento arrojó como resultados que en el clima ético organizacional en las IPS objeto de estudio es relevante la ausencia de la confianza en las relaciones del enfermero con la empresa y con los demás profesionales; su actuar se orienta hacia el respeto a la autoridad, hay heteronomía en la relación con el médico y unas conductas mediadas más por los códigos y señales de la profesión que por las señales organizacionales. La responsabilidad de empleados con la empresa y los pacientes es mayor que aquella de la institución con los empleados. Se sugiere investigar en mayor profundidad si las conductas consideradas "responsables" obedecen a una necesidad de cumplir con la obligación legal o si se trata de una genuina responsabilidad moral.

Palabras Clave: Estatus Ético, percepción, Hospitales. 


\section{Abstract}

In order to measure the nursing-perceived ethics status prevailing in three Health Service Providers HSP in Bogota, a two-stage project was performed. In the first stage, Dr. Linda Olson's designed perception instrument "Ethics Status Perception in Hospitals" was adjusted according to Gutierrez suggestions to include new items applicable to the Colombian context, and to re-asses its reliability.

In the second stage, the adjusted instrument was applied to 219 nurses from three HSP: one public, and one special.

The reliability of the new instrument included a Cronback's alpha of 0.87 , a validity index of 0.66, a trustworthiness index of 0.94 , and an inter-item correlation of 0.38 .

Accounting for $60.22 \%$ of the total variance, eigth factors were obtained.
The corresponding statistics showed that the adjusted instrument is highly reliable to measure the ethics status in hospitals, and that it could be usedas an organizational diagnostic tool.

A HSP nursing-perception finding was the lack of confidence among nurses, hospitals, and other professionals. They perceived that organizational ethics are oriented towards respect to the authority, unilateral commanding, and behavior mediated, more by professional codes, than by organizational signal. They also perceived that more responsibility on patients is deposited on nurses than on other institutional employees.

It is suggested to further insight if the "responsible" conducts arise from a legal obligation or from a genuine moral attitude.

Key Words. Status Ethics, perception, hospitals.

\section{INTRODUCCIÓN}

Aunque hay un creciente interés en las universidades colombianas por la ética y la bioética así como valiosos y numerosos esfuerzos por afianzarla al interior de ellas, la escasa formación en este sentido ha impedido un mayor desarrollo de la reflexión ética en la mayoría de instituciones universitarias en un momento en el que otros valores, como los económicos, parecen estar tomando la supremacía en la toma de decisiones de los profesionales de salud. Esto impone la necesidad de medir el clima ético hospitalario.

Pero; ¿de qué se habla cuando se hace referencia a Clima Etico? Victor y Cullen ${ }^{1}$ definen el Clima Ético Laboral como las percepciones prevalentes de las prácticas y procedimientos organizacionales típicos que tienen un contenido ético. Los autores respaldan su definición en estudios previos de otros psicólogos, que reconocen que, en términos de desarrollo moral, solo las características individuales no explican el comportamiento ético y moral, de lo cual parte la preocupación por el impacto que los factores sociales tienen sobre el individuo.

La existencia de un clima ético laboral implica que se hayan institucionalizado unos sistemas normativos en las organizaciones, que son un elemento de la cultura organizacional. Esto significa, que los miembros de una organización deben percibir que existen unas tendencias normativas dentro de la organización que podrían medirse con cierto grado de consenso. No se trata pues de evaluar los valores o principios de un individuo sino las prácticas y procedimientos que él percibe se desarrollan en su organización. 2,3,4,5

Pocos son los estudios que se han realizado sobre clima ético laboral en general ${ }^{6,7}$ y en el sector salud en particular., ${ }^{8,9}$
La enfermera Linda Olson ${ }^{10}$ diseñó y aplicó un instrumento para medir el Clima Etico Hospitalario $(\mathrm{CEH})$ según la percepción de las enfermeras y evaluar sus propiedades psicométricas. Dicho instrumento fue aplicado a 360 enfermeras de hospitales de Estados Unidos. La validez de constructo fue evaluada usando análisis factorial. El modelo final consistió en 26 variables agrupadas en 5 factores organizados de acuerdo con las relaciones de las enfermeras con sus colegas (4 items), los pacientes (4 items), los administradores/gerentes (6 items), el hospital (6 items) y los médicos (6 items). El alpha de Cronbach del instrumento total fue de 0.91 considerándose la validez y confiabilidad del instrumento aceptables.

Oztürk y Bahcecik ${ }^{11}$ desarrollaron un estudio para evaluar la validez y la confiabilidad del instrumento desarrollado por Olson en Turquía y para determinar cómo perciben las enfermeras el clima ético en su ambiente de trabajo. El estudio fue hecho con 276 enfermeras que trabajaban en universidades, seguridad social, el estado y los hospitales privados. Los investigadores encontraron diferencias significativas que mostraron que el tipo de hospital afecta significativamente el nivel de percepción positiva, así como la edad de la enfermera y la unidad en la que trabajaba.

Con respecto a la validez de la escala en términos estructurales, Bahcecik y Oztürk encontraron que la escala era válida, puesto que cada uno de sus ítems tuvieron un peso del factor por encima de 0.22 , de manera que ninguno de los ítems se excluyó. Aunque la mayoría de las distribuciones de los ítems en subfactores fueron compatibles, ciertos ítem mostraron diferenciación numérica. Los autores concluye- 
ron, a partir de estos hallazgos, que sería mejor emplear la escala como un todo que usar los ítems separadamente.

En el caso colombiano en particular, en el año 2.000 Gutiérrez $^{12}$ realizó un estudio basado en el trabajo publicado por Olson, al cual aplicó pruebas psicométricas de validez y confiabilidad en el contexto colombiano, cuyos resultados señalaron que el instrumento tiene un alto criterio de validez aparente, validez de contenido y consistencia interna con un Alpha de Cronbach de 0.89 .

Sin embargo, este instrumento, en concepto de los investigadores, deja de lado los valores éticos que se consideran pieza fundamental para medir este concepto. Adicionalmente, la definición de Olson de Clima Etico Hospitalario como "condiciones organizacionales y prácticas que afectan la forma como se discuten y deciden los cuidados que requieren los pacientes y que tienen implicaciones éticas" reduce el clima ético a aquel que se evidencia a partir de la relación de los profesionales de salud con el paciente o de las relaciones de ellos entre sí, pero orientadas solo a las decisiones que tienen efecto sobre los pacientes, concepto más limitado que el de clima ético laboral presentado por Victor y Cullen.

\section{Abordaje Metodológico}

En primer lugar, el grupo revisó cada una de las afirmaciones plasmadas en el instrumento original de Olson versión en español e incorporó a este las sugerencias planteadas por Gutiérrez. Se observó en detalle la traducción de la prueba y se mejoró la redacción, especialmente en aquellas afirmaciones que presentaron alpha de Cronbach más bajos en ese estudio (items 2 y 22).

Después de una amplia y profunda revisión bibliográfica el grupo revisó aclaraciones conceptuales acerca de la Ética civil, Ética mínima y ética comunicativa, conceptos que sirvieron como fundamento y análisis para la elaboración de afirmaciones sobre tres valores relevantes para el sistema de salud colombiano a juicio de los investigadores: Honestidad ${ }^{13,14,15}$ Responsabilidad ${ }^{16}$ y Tolerancia ${ }^{17,18,19,20}$. El grupo investigador ayudó a determinar la validez aparente del cuestionario y su validez de contenido fue revisada por 7 jueces expertos en ética y/o bioética, quienes valoraron su pertinencia, relevancia y comprensión y la comprensión de las primeras 26 afirmaciones de la prueba de Olson. Luego se realizó un pilotaje con 45 enfermeras de diferentes hospitales para medir consistencia interna.

En una segunda fase, el instrumento fue aplicado a 219 enfermeras en tres instituciones hospitalarias de Bogotá:
Una de carácter público, otra de carácter privado y una tercera correspondiente a un régimen especial de salud de las Fuerzas Militares. Se solicitó autorización a las directivas de cada una de las instituciones y sólo se aplicó a enfermeros profesionales con al menos tres meses de antigüedad en la institución que voluntariamente quisieran responderlo. Se mantuvo la confidencialidad de las instituciones colaboradoras y el grupo se comprometió a socializar con cada una los resultados correspondientes a esa entidad

La información recopilada fue incorporada en SPSS (Versión 10) para realizar los análisis de confiabilidad entre las escalas y de validez del cuestionario global.

Para determinar la confiabilidad se realizó el análisis de la consistencia interna a través del alfa de Cronbach. Para establecer la validez de constructo, se efectuó un análisis factorial usando el método Varimax, que permitió hallar el mínimo de grupos o factores que subyacen a toda la prueba y que explican la mayor cantidad de varianza en la misma, lo cual indica que está midiendo un mismo concepto o constructo teórico.

\section{RESULTADOS Y DISCUSIÓN}

En relación con el ajuste de las preguntas del cuestionario de Olson que revisó Gutierrez y de acuerdo con sus sugerencias, se modificaron 17 de las 25 preguntas elaboradas. Posteriormente se elaboraron las preguntas relacionadas con los valores de honestidad, responsabilidad y tolerancia, de las cuales finalmente quedaron 15:4 de responsabilidad, 7 de honestidad y 4 de tolerancia.

Las afirmaciones que presentaron problemas de comprensión fueron ajustadas. El cuestionario entonces quedó conformado por 41 afirmaciones con una escala de respuesta de 1 a 4 , siendo $1=$ Nunca, 2 = Algunas veces, $3=$ Con frecuencia y $4=$ Siempre.

En la aplicación de la prueba piloto a 45 enfermeros profesionales, se usó el Alpha de Cronbach para verificar la confiabilidad de las 41 afirmaciones, obteniéndose una correlación de 0.894 para el cuestionario total.

El alpha de Cronbach para las afirmaciones de Olson fue de 0.86 y de 0.74 entre las nuevas afirmaciones obteniendo una menor correlación para la afirmación No 11 (Al paciente se le trata de acuerdo a la ARS, EPS o prepagada a la que estén afiliados), que fue eliminada, quedando un instrumento conformado por 40 items.

Corregido el instrumento a partir de los hallazgos de la prueba piloto se realizó el análisis de la confiabilidad global del cuestionario con 40 ítems que se aplicó a 219 enferme- 
ros de los tres hospitales objeto de estudio. Las encuestas con más del $10 \%$ de omisiones no se tuvo en cuenta, quedando así 209 encuestas para los análisis, de los cuales se obtuvo un alpha de Cronbach de 0.94 entre todas las afirmaciones.

El alpha de Cronbach total (40 items) fue de 0.94. Al analizar por aparte los 14 items relacionados con valores, se obtuvo un alpha de Cronbach de 0.87 y de 0.92 para los 26 items de la encuesta de Olson. En la Tabla 1 se observa que los índices de consistencia interna para cada una de las categorías que mide el cuestionario son inferiores al de la prueba como un todo.

Para medir la validez de constucto se hizo un análisis factorial, como se observa en la tabla 2: Los primeros 8 factores obtenidos explicaron el $60.22 \%$ de la varianza. El grupo analizó los contenidos de los ítems agrupados en cada factor y lo rotuló de acuerdo con el concepto teórico. Así, los ítems de las 8 categoías se agruparon en 7 factores: el respaldo (sentido por los clientes internos de la institución), el reconocimiento del paciente como sujeto de derechos, la confianza, la responsabilidad, el compañerismo, el respeto y la comunicación.

En relación con los resultados del cuestionario aplicado a 219 enfermeras, se encontró que el 38\% con frecuencia y el $25 \%$ siempre perciben que el siempre ayuda cuando los colegas son incapaces de tomar decisiones por sí solos. Sin embargo, el $32 \%$ sugiere que falta colegaje en cuanto a la escucha y $36 \%$ (78) en la ayuda frente a los problemas relacionados con el cuidado del paciente; un 37\% (81) percibe falta de colegaje tanto de sus colegas como de su superior.

En relación con la competencia profesional el $80 \%$ de los enfermeros considera que trabaja con colegas competentes siempre o frecuentemente y un $20 \%$ opina que esto ocurre nunca o casi nunca. En este sentido cabe preguntarse bajo qué criterio se está valorando la competencia profesional.

Respecto al uso de la información para el cuidado del paciente, los resultados sugieren que teniendo el acceso a ella ( $80 \%$ de las enfermeras respondieron que siempre o con frecuencia tienen acceso a ella), algunas enfermeras no aprovechan este recurso para la toma decisiones (17\% dice acceder "algunas veces" o "nunca").

Los resultados que hacen referencia a los derechos de los pacientes, como por ejemplo, que sus hábitos sean tenidos en cuenta, la información que se les da sobre los riesgos y beneficios de las intervenciones, entre otros, parecen dar cuenta de que la protección de los derechos de los pacientes están mediados por la relación contractual que impone el sistema porque la relación contractual así lo obliga (ejemplo, consentimiento informado). En este sentido, se protegen los derechos que cobija el "contrato" de prestación de servicios o el "seguro" pero queda la duda de si se respetan porque genuinamente hay un interés por proteger la vida y por respetar la dignidad de la persona, o si esto deja de ocurrir porque el contrato no lo cubre. Particularmente la información que se da a los pacientes sobre sus derechos puede referirse a la confirmación de aquello a que tienen derecho cuando este formula una pregunta al respecto o cuando la enfermera considera que está siendo vulnerado el derecho.

En la categoría administradores los resultados son contradictorios: el $96 \%$ de los encuestados dicen respetar a su jefe y en las afirmaciones relacionadas con la confianza que genera el jefe, su capacidad de escucha, entre otras, no superó el 48\% para las afirmaciones de siempre, lo cual refleja que el respeto no está basado en la confianza, sino en la autoridad que confiere el cargo, lo cual podría traducirse en sumisión, más aun en las condiciones de incertidumbre laboral actual.

Con respecto a la toma de decisiones del cuidado del paciente se observa que el jefe inmediato insta a la enfermera a que tome las decisiones por sí misma, sin darle apoyo en un $28 \%$.

En los resultados de categoría de hospital 75\% destacó la afirmación "en mi servicio practico la enfermería como creo que debe practicarse". En este sentido, las enfermeras se comportan más como profesionales de enfermería que como miembros de la empresa.

En la relación del enfermero y el médico se observa que el $48 \%$ de los encuestados considera que nunca o algunas veces hay una relación profesional basada en la confianza, que se mantiene una asimetría en esta relación, en donde el médico es autoridad frente al enfermero, y en donde la heteronomía parece hacerse visible; en tanto que el 59\% de los encuestados considera que hay respeto del médico hacia la opinión del enfermero algunas veces o nunca. El respeto del médico hacia el enfermero se basa entonces en el reconocimiento de éste como persona, más no como profesional.

Por otra parte, el $46 \%$ de los enfermeros no siempre siente respaldo de la institución misma; en este sentido, no percibe reconocimiento en el entorno laboral.

Hay una contradicción en relación con la responsabilidad, que parece hacerse más visible en particular cuando 
se trata de hacerse responsable de las consecuencias de los actos. La aplicación de protocolos podría obedecer a la obligación legal.

En cuanto al valor de la honestidad, el 59\% de los encuestados considera que la institución no reconoce nunca los aciertos y desaciertos de sus trabajadores sin privilegios laborales (14\%) o lo hace algunas veces (45\%). Sin embargo, la percepción de la honestidad de la empresa en términos de lo que comunica a los pacientes es positiva en un $52 \%$, puesto que con frecuencia se informa a los pacientes sobre su situación de salud, los riesgos y beneficios de las intervenciones y se mantiene fidelidad en los registros. Habría que preguntarse si la honestidad que se expresa en estas afirmaciones, es un valor por sí mismo o si estas conductas obedecen a la necesidad de cumplir las normas porque son exigencia externa o de cumplirlas porque de no hacerlo pueden afectar a la empresa económicamente (p.e.: a través de demandas).

En cuanto a la tolerancia este es un aspecto que no muestra resultados sobresalientes, a excepción del relacionado con el hecho de que para el 38\% de los encuestados la institución no tiene en cuenta las sugerencias que hacen los clientes externos e internos, lo cual confirma el planteamiento de que el interés de la empresa no se centra en los empleados ni en los usuarios para este porcentaje de encuestados.

El $71 \%$ de los enfermeros considera que al paciente le son respetados los hábitos para su cuidado y $69 \%$ de ellos considera que se permite la intervención de la familia, aspecto muy positivo para fortalecer la atención integral en el cuidado.
Por otra parte se concluye que es mayor la responsabilidad de los empleados con los pacientes y con la empresa, y de la empresa con los pacientes que de la empresa con sus empleados, lo cual influye directamente en el clima organizacional en general.

A pesar de que se observa en las instituciones la definición de una plataforma estratégica, para un importante porcentaje de encuestados no hay un direccionamiento claro desde las instancias directivas de la institución hacia sus empleados, que orienten el quehacer institucional y permitan que prevalezca una cultura organizacional claramente visible.

\section{CONCLUSIONES}

Se encontró que la encuesta vista de manera global, tiene una confiabilidad alta, indicando que está midiendo internamente un mismo aspecto. En cuanto a la validez de constructo, se encuentra que, si bien no resultaron los factores de acuerdo con las categorías o valores que pretende medir, sí se encuentran emergiendo factores que explican conceptos importantes dentro del $\mathrm{CEH}$.

De la aplicación del instrumento en las instituciones objeto de estudio se puede concluir que en el clima organizacional en estas instituciones es relevante la ausencia de la confianza en las relaciones del enfermero con la empresa y con los demás profesionales (Tabla 3); el actuar del enfermero se orienta hacia el respeto a la autoridad, hay heteronomía en la relación con el médico y conductas mediadas más por los códigos y señales de la profesión que por las señales organizacionales. La responsabilidad de empleados con la empresa y los pacientes es mayor que aquella de la institución con los empleados. (Tabla 4).

\section{Resultados validez de contenido}

\begin{tabular}{|c|c|c|c|c|c|c|} 
Afirmación & Valor & Pertinencia & Relevancia & Comprensión & Promedio & Porcentaje \\
\hline \hline 1 & Responsabilidad & 6 & 7 & 5 & 6 & 0.86 \\
\hline 2 & Honestidad & 7 & 7 & 7 & 7 & 1.00 \\
\hline 3 & Responsabilidad & 6 & 7 & 5 & 6 & 0.86 \\
\hline 4 & Honestidad & 6 & 6 & 2 & 4.66 & 0.66 \\
\hline 5 & Tolerancia & 6 & 6 & 6 & 6 & 0.86 \\
\hline 6 & Tolerancia & 7 & 7 & 7 & 7 & 1.00 \\
\hline 7 & Tolerancia & 6 & 5 & 4 & 5 & 0.71 \\
\hline 8 & Responsabilidad & 6 & 6 & 4 & 5.33 & 0.76 \\
\hline 9 & Honestidad & 7 & 7 & 4 & 6 & 0.86 \\
\hline 10 & Honestidad & 7 & 7 & 7 & 7 & 1.00 \\
\hline 11 & Honestidad & 4 & 5 & 5 & 4.66 & 0.66 \\
\hline 12 & Tolerancia & 6 & 6 & 6 & 6 & 0.86 \\
\hline 13 & Honestidad & 7 & 7 & 6 & 6.6 & 0.95 \\
\hline 14 & Honestidad & 7 & 7 & 7 & 7 & 1.00 \\
\hline 15 & Responsabilidad & 7 & 7 & 5 & 6.33 & 0.90 \\
\hline \hline
\end{tabular}


Puntajes Alfa para las categorías de la ECEH en la prueba definitiva

\begin{tabular}{|c|c|c|c|}
\hline \multirow{2}{*}{ Categoría/Valor } & \multicolumn{2}{|c|}{ Correlación ítem-escala } & \multirow{2}{*}{$\begin{array}{c}\text { Alfa de } \\
\text { Cronbach }\end{array}$} \\
\cline { 2 - 3 } & Mínimo & Máximo & 0.66 \\
\hline Colegas & 0.40 & 0.48 & 0.65 \\
\hline Paciente & 0.34 & 0.52 & 0.86 \\
\hline Administradores & 0.40 & 0.78 & 0.73 \\
\hline Hospital & 0.20 & 0.61 & 0.81 \\
\hline Médicos & 0.51 & 0.68 & 0.69 \\
\hline Responsabilidad & 0.40 & 0.53 & 0.75 \\
\hline Honestidad & 0.35 & 0.57 & 0.67 \\
\hline Tolerancia & 0.36 & 0.49 & \\
\hline \hline
\end{tabular}

Ítems por categoria de la encuesta que cargan en los ocho factores extraidos

\begin{tabular}{|c|cccccccc|}
\cline { 2 - 9 } \multicolumn{1}{c|}{} & \multicolumn{7}{c|}{ FACTORES } \\
\hline $\begin{array}{c}\text { Categoria 0 } \\
\text { Valor }\end{array}$ & $\mathbf{1}$ & $\mathbf{2}$ & $\mathbf{3}$ & $\mathbf{4}$ & $\mathbf{5}$ & $\mathbf{6}$ & $\mathbf{7}$ & $\mathbf{8}$ \\
\hline Colegas & 2 & 1 & 1 & & 2 & 2 & & 1 \\
Paciente & & 1 & & 2 & 3 & & & 1 \\
Administradores & 5 & & & & & 1 & 1 & \\
Hospital & 4 & 1 & 1 & 2 & 1 & & 1 & \\
Médicos & 1 & & 3 & 5 & & 1 & & 1 \\
Responsabilidad & & 2 & 1 & & 2 & & & \\
Honestidad & & 4 & 3 & & 1 & 1 & 1 & $1(-)^{*}$ \\
Tolerancia & 1 & 3 & 1 & & & 1 & & 1 \\
\hline TOTAL & $\mathbf{1 3}$ & $\mathbf{1 2}$ & $\mathbf{1 0}$ & $\mathbf{9}$ & $\mathbf{9}$ & $\mathbf{6}$ & $\mathbf{3}$ & $\mathbf{5}$
\end{tabular}

*NOTA: Este item fue el único que obtuvo una carga negativa dentro del factor 8 ( $\mathrm{y}$ a lo largo de los factores obtenidos) lo cual hace que exista bipolaridad en este factor.

Es prioritario seguir trabajando a nivel de las instituciones hospitalarias y en las instituciones que forman enfermeros en la conceptualización y formación en valores.

Se sugiere investigar en mayor profundidad si las conductas consideradas "responsables" obedecen a una necesidad de cumplir con la obligación legal o si se trata de una genuina responsabilidad moral.

\section{RERERENCIAS BIBLIOGRÁFICAS}

1 Victor B, Cullen JB. The organizational bases of Ethical Work climates. Administrative Science Quarterly. 1988; 33(1): 101-125.

2 Cortina A. Etica de la empresa. Capítulo 4. Editorial Trotta. Madrid, España. 1996. p. 86.

3 Koontz H, Weihrich H.. Administración, Una perspectiva global. McGrawHill. 11 edición. México.1998. p.69.

4 Hill CWL, Jones GR. Administración Estratégica. McGrawHill. Colombia. 1998. P. 57

5 Franca-Tarragó O. La ética al interior de las empresas. En:

http://www.ucu.edu.uy/Facultades/CienciasHumanas/Departamentos/Etica/Publicaciones/ 3Etica\%20Socioeconomica/MACLIMAeti.doc.

6 Ariño A, Conill J, García D, Garrido E, González E, Llofríu M, Soriano C. La dimensión ética de la cultura empresarial en la Comunidad Valenciana. Fundación Etnor. En: Revista Economía 3. Julio-agosto 2000. En: www.etnor.org/ladimensionetica.pdf
7 Fontrodona J, De los Santos J. Clima ético de la empresa española: Grado de implantación de Prácticas Eticas. IESE Business School, Universidad de Navarra. 2004. En: http:// ideas.repec.org/p/ebg/iesewp/d-0538.html

8 Bell SE. Ethical climate in Manager Care Organizations. Nursing Administration Quarterly. 2003; 27(2):133 . Lippincott Williams \& Wilkins, Inc.

9 Hart SE. Hospital ethical climates and registered nurses 'turnover intentions. Journal of Nursing Scholarship. Sigma Theta Tau Internacional Honor Society. 2005; 37(2); 173-177.

10 Olson, L. Journal of Nursing Scholarship. Saint Xavier University, Chicago, Illinois 1998; 30(4): 345-349.

11 Ozturk H.; Bahcecik, N. The hospital Ethical Climate survey in Turkey. Journal of Nursing Administration. Healthcare Law, Ethics and Regulation. 2003. Vol. 5 (4) pp. 94-99.

12 Gutierrez C. Validez aparente, validez de contenido y consistencia interna de la Encuesta de Clima Etico Hospitalario. Maestría en Enfermería, Facultad de Enfermería. Universidad Nacional de Colombia. Bogotá, 2001.

13 Morales A. Monografía: "Ios valores humanos". En: www. portalplanetasedna.com.cu/valores, htm

14 Rumbold G. Ética en Enfermería. Traducción Ma. Teresa Aguilar. Editorial McGraw-Hill Interamericana, 3a edición, México, 2000

15 Woodstock Theological Center. Creating and mantaining an ethical corporate climate. Georgetown University Press. 1990. En: woodstock.georgetown.edu/programs/wbp/business_ethics/cmecc.htm

16 Arango G. Responsabilidad moral y responsabilidad profesional. En imprenta.

17 Asamblea General, Organización de las Naciones Unidas. Declaración del Milenio Resolución aprobada por la Asamblea General. [sin remisión previa a una Comisión Principal (A/55/L.2)]55/2. Septiembre 8 de 2000.

18 Vernon R, LaSelva SV. Justifying tolerance. Canadian Journal of Political Science. Canadá. 7(1) Marzo. 1984. p. 3-23.

19 Devlin, Patrick. The Enforcement of Morals. Patrick Devlin, The Enforcement of Morals Oxford University Press. 1965.

20 López Crestar, JR. Tolerancia e indiferencia. Aclarando un concepto muy usado pero generalmente de forma errónea, por malicia o por ignorancia. Revista Arbil n 77 . En: http:// www.arbil.org/(77)tole.htm

\section{DIRECCIÓN PARA CORRESPONDENCIA}

Gloria Inés Romano Prieto: gloriaromano@etb.net.com 